\title{
CHRONIQUE
}

\section{Utilisation des eaux usées urbaines pour l'élevage des Carpes}

Nous exprimons notre vive gratitude à $M$. Goscinski qui a bien voulu nous transmettre l'excellent travail du docteur P. Wolny, sur l'utilisation des eaux résiduaires.

De la Station expérimentale de carpiculture de Zabieniec, près de Varsovie, le docteur Wolny s'est attaché à ce problème. Le volume des eaux usées des grandes villes s'accroût, en effet, chaque année, dans des proportions considérables et son évacuation devient une source de dépenses importantes.

Celles-ci, croyons-nous, devraient pouvoir être sensiblement diminuées par l'utilisation de ces eaux résiduaires à des fins piscicoles ainsi qu'après de pertinentes études le docteur $P$. Wolny nous le démontre.

Nous le remercions donc très vivement de nous avoir autorisé à faire connaître ici ses travaux.

Nos remerciements s'adressent égalemènt à $M^{\text {He }}$ Nisbet, chef de travaux de chimie, gràce à qui cette étúde a pu paraître dans le Bullètin français de pisciculture.

\author{
Hervé de Villette, \\ Président de l'Union nationale \\ des Syndicats de l'Étang.
}

Les méthodes biologiques d'épuration des égouts urbains permettent de transformer les matières organiques contenues dans ces égouts en sels minéraux d'azote, de phosphiore et de patassium. Nous déversons journellement dans les rivières plus de $200.000 \mathrm{~m}^{3}$ d'eau d'égout épurée provenant de différentes. stations d'épuration urbaines. Et de nouvelles stations de ce type sont prévues dans le plan d'urbanisme du proèhe avenir.

La quantité d'eau d'égout indiquée ci-dessus suffirait actuellement à remplir et maintenir en eau au moins 500 ha d'étangs de pisciculture. Nous savons qu'à la pisciculture de Mala Rasowa qui reçoit des égouts purifiés de la ville de Legnica, ces égouts sont dilués, avec l'eau du torrent avant l'introduction dans les étangs. Les rendements qui atteignaient en $1958,490 \mathrm{~kg} / \mathrm{ha}$ pour les alevins (nourriture naturelle) et $1.100 \mathrm{~kg} / \mathrm{ha}$ pour les Carpes bisannuelles 
(avec alimentation complémentaire) démontrent la grande valeur des eaux d'égouts utilisées comme engrais. Mais dans notre pays, et particulièrement dans le Centre où la pénurie d'eau se fait sentir en été, l'utilisation des eaux d'égouts comme engrais se trouverait limitee par manque d'eau de dilution.

Voulant savoir maintenant si les eaux d'égouts épurées pouvaient être utilisées sans dilution, des études ont été effectuées pendant 4 ans (1958-1961), dans cinq étangs d'une superficie de 321 à $1.806 \mathrm{~m}^{2}$, alimentés par les eaux de la - stạtion-d'épuration de Kielce. Des études ont porté sur l'élevage des Carpes, Carassins, Tanches et Sandres. Pour les Carpes, des essais ont été poursuivis en

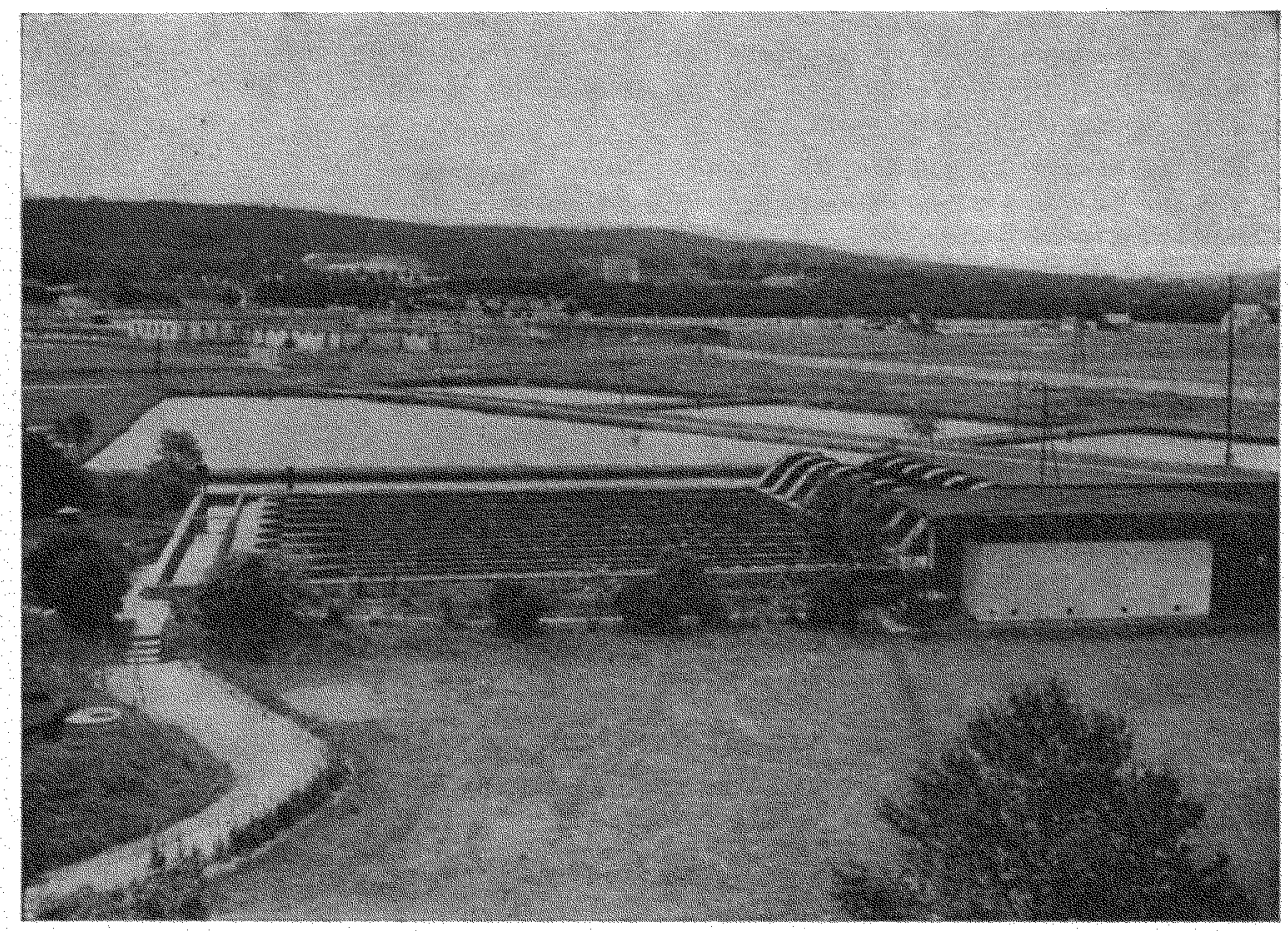

Station d'épuration de Kielce

hiver pour connaitre le comportement des alevins pendant l'hivernage dans ces eaux d'égout épurées par le procédé des boues activées.

Au point de vue physique, ces eaux d'égouts épurées correspondent à une eau de rivière : elles sont presque claires, incolores et sans odeur. Cependant les concentrations en sels minéraux sont toujours beaucoup plus élevées que celles de la rivière voisine (Jeziorka). En janvier 1960, par exemple, l'eau d'égout épurée contenait $44 \mathrm{mg} / \mathrm{l}$ d'azote nitrique, alors qu'il n'y en avait que $3,0 \mathrm{mg}$ 'l dans la rivière. De mème pour les phosphates, elément nutritif indispensable pour les plantes : on trouvait $2,5 \mathrm{mg} / \mathrm{l}$ de phosphore dans l'eau d'égout contre 0,07 dans l'eau de rivière. En été, la concentration en azote et phosphore dans les étangs de Kielce était environ quatre fois moins grande, mais restait encore très supérieure aux concentrations trouvèes généralement dans les piscicultures. Ces grandes quantités de sels minéraux ont eu une influence favorable sur le développement des plantes aquatiques. 
Les observations faites pendant quatre ans sur ces étangs ont montré qu'un peuplement relativement important en poissons accélère le développement du phytoplancton et diminue celui des algues filamenteuses, des mousses et des plantes vasculaires du fond des étangs. En effet, l'ensemble de ces plantes recouvre en une seule saison $70 \%$ des étangs de contrôle, vides de poissons alors que les étangs peuplés sont restés pendant 4 ans sans végétation : les Carpes en se nourrissant arrachaient les pousses au fur et à mesure de leur sortie; en même temps, l'eau devenue trouble faisait obstacle aux rayons solaires et entravait ainsi la croissance des algues filamenteuses et des végétaux. Par contre, les poissons n'ont aucune influence défavorable sur la croissance des plantes aquatiques flottantes.

Au point de vue phytoplancton, on a pu constater que les espèces n'étaient pas les mèmes dans lés étangs peuplés et dans les étangs de contrôle. I)ans ces derniers, dominent les grandes formes d'Oscillatoria et les diatomées du genre Melosira. Dans les étangs empoissonnés apparaît dès le début du printemps, une intense couleur vert émeraude due à un plancton à chlorophycées qui se maintient constamment jusqu'à la vidange des étangs à l'automne. Ce plancton à chlorophycées comprend surtout des espèces des genres Scenedesmus et Chlorella. Dans un des étangs peuplés, on a établi un courant d'eau important pour connaître l'influence du courant. Dans cet étang, il n'y a pas eu de développement planctonique visible car le courant entrainait le plancton des sa formation. De plus, dans cet étang sont apparues les algues filamenteuses et les plantes vasculaires malgré la présence de poissons. Donc l'engrais naturel apporté par les eaux d'égouts épurées produit des fleurs d'eau permanentes uniquement dans les étangs peuplés et en eau stagnante (ou alimentés par un très faible courant d'eau permettant au plancton de se multiplier). On sait que le cycle de développement de ces chlorophycées est de 8 à 10 jours.

A la suite de ces observations, on pouvait prevoir une production doxygène importante et durable dans les étangs alevinés ct sans courant.

Si l'on admet l'oxygène comme facteur limitant l'intensité d'alimentation des Carpes, le maximum diurne en surface et le minimum nocturne au fond délimitent la zone de pâturage des Carpes.

Il résulte des chiffres du tableau no I que les Carpes des étangs à eau stagnante, contrairement à celles des étangs à eau courante, pouvaient se nourrir à volonté à toute heure du jour ou de la nuit : dans les deux ètangs à eau stagnante (nos 2 et 3) la teneur en oxygène dissous au fond et pendant la nuit n'est jamais descendue au-dessous de $6,0 \mathrm{mg} / \mathrm{l}$. C'est pourquoi on ne doit pas craindre un déficit en oxygène en été dans des étangs peuplés, stagnants, irrigués exclusivement avec des eaux d'égouts épurées. Pendant l'été 1961, il n'y eut qu'un déficit de courte durée dans un étang dont la surface était recouverte de lentille d'eau à $90 \%$ et empoissonné de nourrains de Carpe.

Le phytoplancton crée une base nutritive pour les invertébrés : les Daphnidés, les Copépodes et les Rotifères se nourrissent de plancton vivant, tandis que la faune de fond se nourrit des cellules mortes tombant au fond des ètangs.

Une étude des invertébrés de fond dans les étangs peuplés de Carpes de deux étés et irrigués avec des égouts urbains épurés, a montré que le peuplement était environ 3 fois plus important que le peuplement moyen des étangs de pisciculture en Pologne. Parmi ces invertébrés de fond, ce sont les larves de Tendipedidés (élément essentiel de l'alimentation naturelle des Carpes) qui dominaient dans 13 échantillons sur 15. 


\section{D - Doprowatzolnk}

\section{Staw zarybiony (przeplyw)}
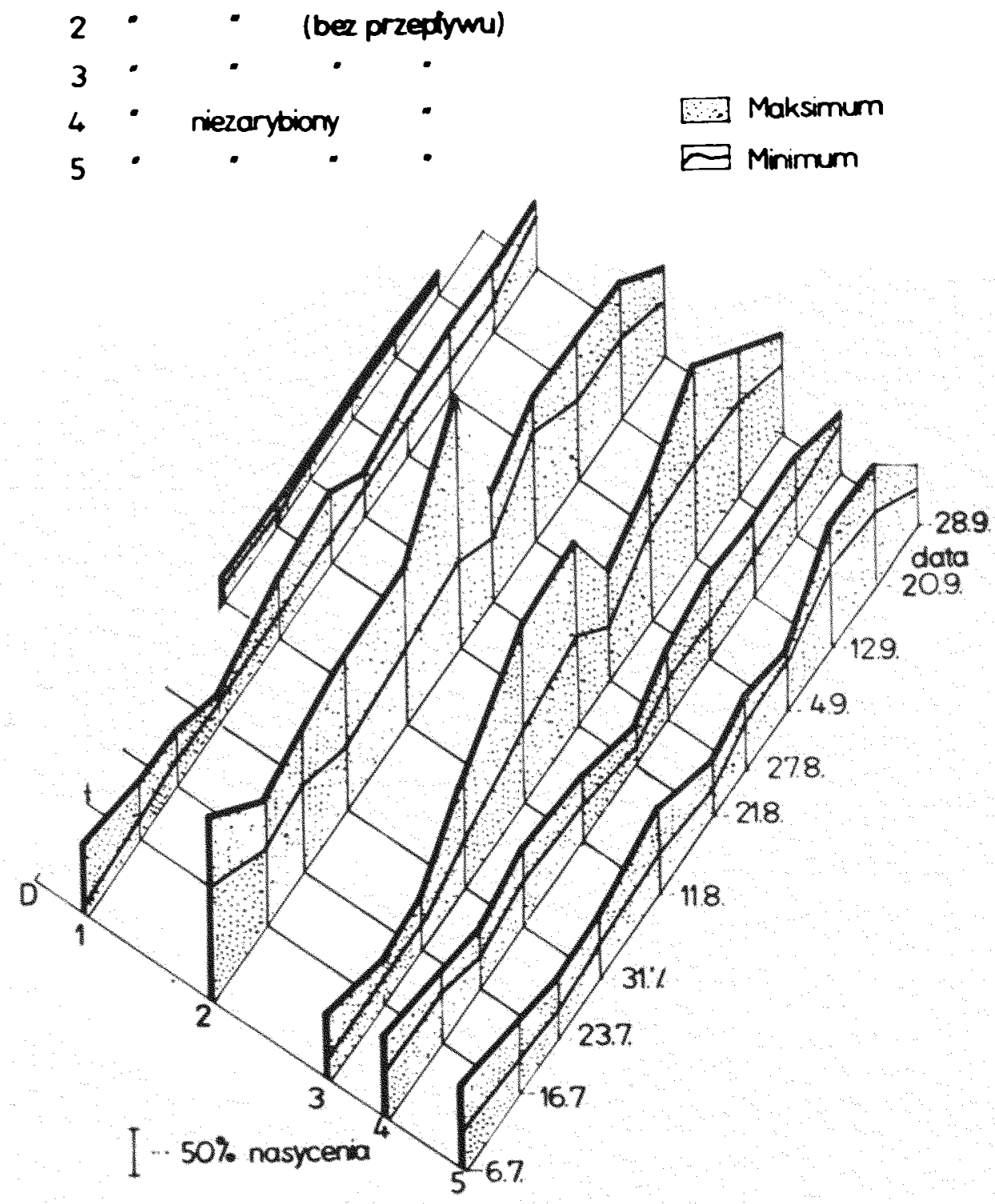

Concentration en oxygène dissous dans les étangs peuplés de Carpes et dans les étangs de contrôle, vides_de poissons.

Tous les étangs sont alimentés exclusivement avec.des eaux d'égouts purifiées. Kielce, 1959.

D- Canal d'alimentation.

1 - Etang peuplé (eau courante).

2 - Etang peuplé (eau stagnante).
3 - Etang peuplé (eau stagnante).

4 - Etang de contrôle.

5 - Etang de contrôle.

$$
\text { nasycena }=\text { saturation } \quad \text { data }={ }_{-}^{-} \text {dates }
$$




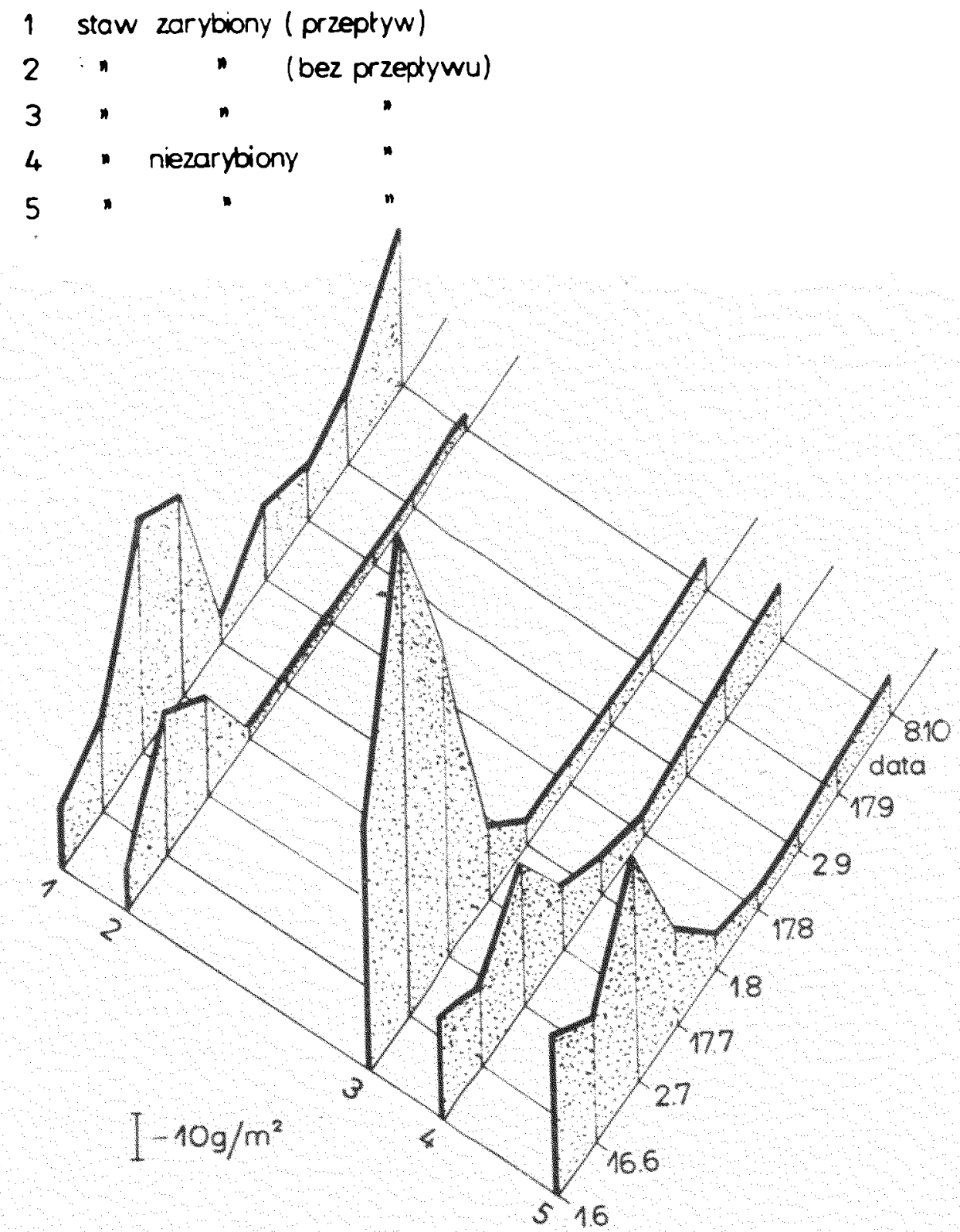

Dynamique de la Biomasse des Tendipedidés dans les étangs alimentés par des égouts épurés.

Kielce, 1959.

1 - Etang en charge (eau courante).

2 - Etang en charge (eau stagnante).

3 - Etang en charge (eall stagnante).
4 - Etang de contrôle (eau stugnante).

5 - Étang de contrôle (suns poisson). 
Par conséquent les deux facteurs : quantité d'oxygène dissous et faune nutritive abondante, fournissent aux poissons des conditions très favorables.

L'étude des contenus intestinaux des Carpes confirme cette affirmation : malgré la densité du peuplement, les Carpes avaient pendant toute la saison, des tubes digestifs remplis d'invertébrés. En outre, on a constaté chez lés alevins comme chez les nourrains une prépondérance des Tendipedidés sur les Crustacés. Par exemple, chez les Carpes bisannuelles de l'étang $\mathrm{n}^{\circ} 3$, il y avait

\section{TABLEAU I.}

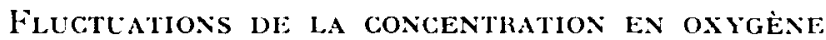

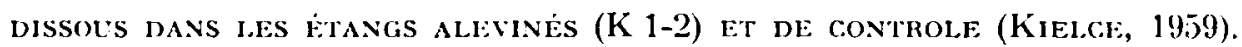

\begin{tabular}{|c|c|c|c|c|c|}
\hline & \multicolumn{3}{|c|}{ Lles ÉTANGS ALENINIS } & \multicolumn{2}{|c|}{$\begin{array}{l}\text { LES ERANiS } \\
\text { SANS Alivins } \\
\text { (de contròle) }\end{array}$} \\
\hline & \multicolumn{2}{|c|}{ En eau stagnante } & \multirow{2}{*}{$\begin{array}{c}\begin{array}{c}\text { En eau } \\
\text { courante }\end{array} \\
1\end{array}$} & \multicolumn{2}{|c|}{ En eau stagnante } \\
\hline \multirow[t]{2}{*}{ No des étangs } & 2 & 3 & & 1 & j) \\
\hline & $\left(\mathrm{mg} \mathrm{O}_{2} / \mathrm{l}\right)$ & $\left(\operatorname{lng}()_{2} / 1\right)$ & $\left(\operatorname{mgg} \mathrm{O}_{2} / \mathrm{l}\right)$ & $\left(\mathrm{mg} \mathrm{O}_{2} / \mathrm{l}\right)$ & $\left(\operatorname{mg}()_{2} / h\right)$ \\
\hline $\begin{array}{l}\text { Maximum diurne } \\
\text { en surface } \ldots . . .\end{array}$ & 14,30 & $11,7^{2}$ & 5,56 & 6,50 & 7,12 \\
\hline $\begin{array}{l}\text { Minim um noc- } \\
\text { turne au fond. }\end{array}$ & 7,69 & 6,27 & $2,6.4$ & 3,17 & 1,57 \\
\hline
\end{tabular}

$57 \%$ de larves de Tendipedidés contre $23 \%$ de Crustacés, le reste étant constituc de débris végétaux et des autres groupes d'animaux.

La croissance des poissons dans ces étangs dépasse de beaucoup celle

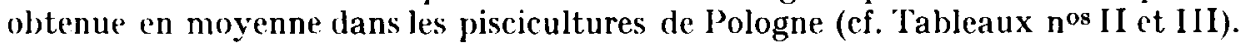
En 1958, avec un alevinage faible au départ ( 7.500 alevins par hectare) et effectue relativement tard (14 Juillet), le rendement s'élève à $469-493 \mathrm{~kg} / \mathrm{ha}$ avec un poids moyen individuel de $140 \mathrm{~g}$. En 1961 , un alevinage plus tardif (27 Juillet) abaissa le rendement à $230 \mathrm{~kg} / \mathrm{ha}$. Fnfin, dans les etangs à eau courante dont le's conditions d'oxygénation sont plus mauvaises, le rendement tombait à 111$1.56 \mathrm{~kg} / \mathrm{ha}$.

De telles différences entre les deux sortes d'étangs (eau courante - eau stagnante) ne s'observent pas avec des poissons de deux étés, plus résistants aux variations d'oxygénation : en 1959, le rendement dans l'étang à eau courante 
TIBILA:Al: 11 .

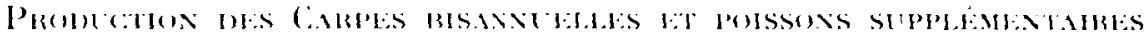
(Rendement en kilogranmes par liectare)

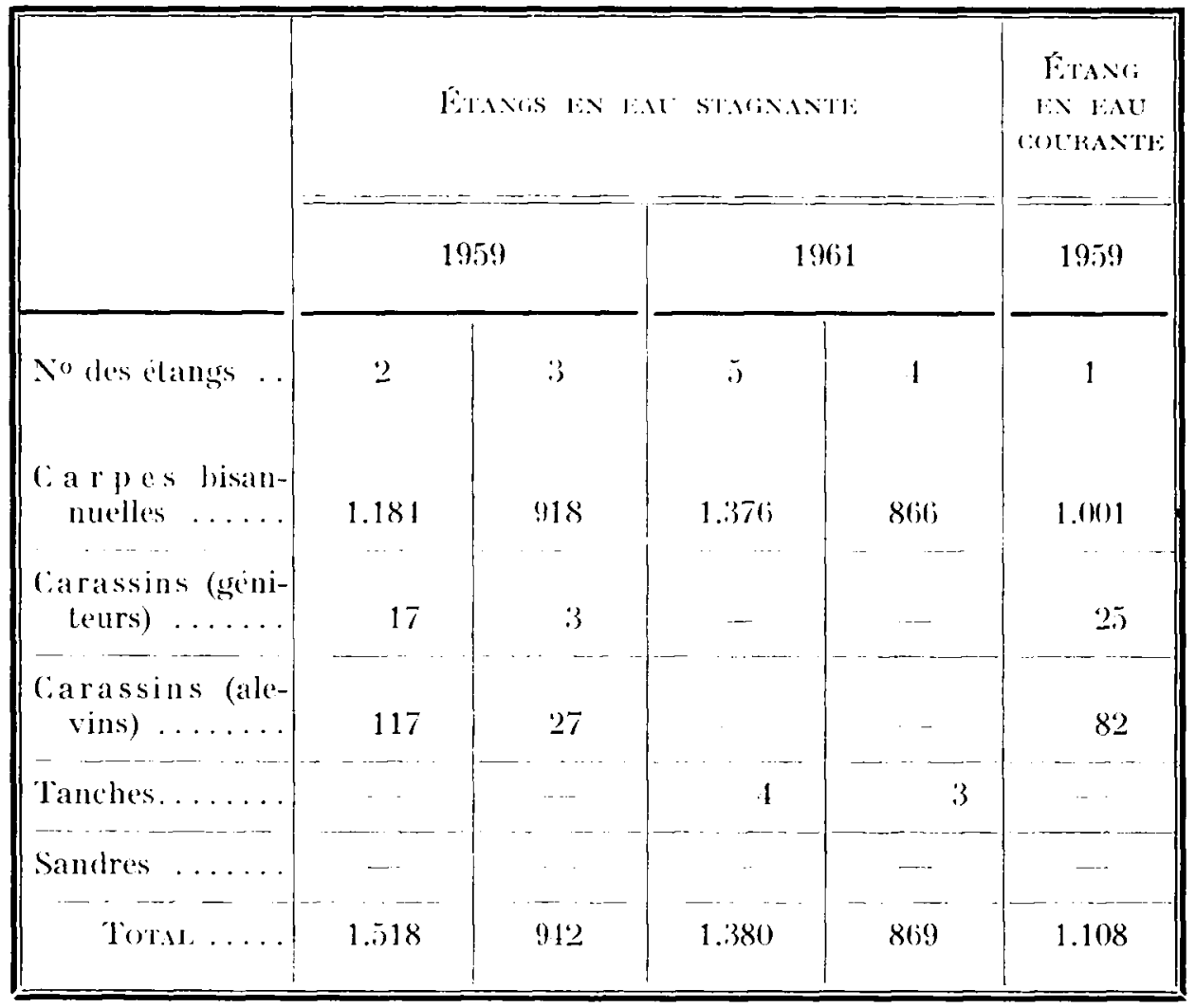

TABILEAU III.

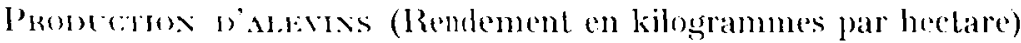

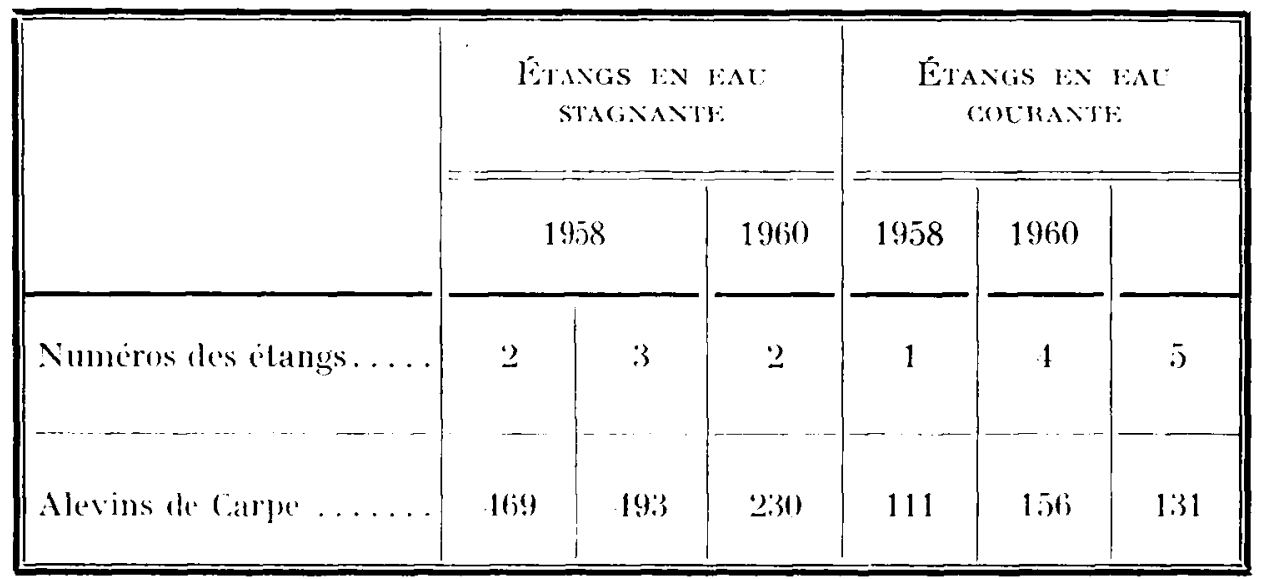


atteignit $1.001 \mathrm{~kg} / \mathrm{ha}$ tandis qu'on obtenait dans les étangs à eau stagnante des rendements de 866 à $1.376 \mathrm{~kg} / \mathrm{ha}$ avec des Carpes de deux ètés. L'alevinage en Sandre n'a rien donné : un seul Sandre fut retrouvé sur ceux introduits en 1961. Par contre, on peut ajouter, à còté des Carpes, des Carrassins dorés et des Tanches.

Des prises d'essai de Carpes de deux étés effectuées systématiquement toutes les deux semaines ont permis de saisir la dynamique de la croissance des poissons pendant la saison active. Lés résultats de ces essais sont donnés dans le tableau $n^{\circ}$ IV en comparaison avec ceux obtenus dans des étangs d'une pisciculture normale, c'est à-dire alimentés par de l'eau de rivière, mais oủ les poissons reçoivent une abondante nourriture artificielle. On peut constater que les taux de croissance sont plus ou moins parallèles. L'abondance de la nourriture naturelle dans

TABLEAU IV.

Taux de croissance des Carpes bisannuelles

\begin{tabular}{|c|c|c|}
\hline $\begin{array}{c}\text { Depuis la date } \\
\text { d'alevinage } \\
\text { (14 Avril 1959) }\end{array}$ & $\begin{array}{c}\text { A KIELCE : } \\
\text { Étang no } 2 \text { empli } \\
\text { avec des égouts épurés }\end{array}$ & $\begin{array}{l}\text { Pisciculiture Wilga : } \\
\text { Étang n }{ }^{\circ} 2 \text { empli } \\
\text { avec de l'eau de rivière }\end{array}$ \\
\hline Jusqu'à la fin de Mai & $7,1 \%$ & $11,4 \%$ \\
\hline Juin & $34,7 \%$ & $21,2 \%$ \\
\hline Juillet ...... & $42,5 \%$ & $29,0 \%$ \\
\hline Août ..... & $10,0 \%$ & $28,1 \%$ \\
\hline Septembre ... & $5,7 \%$ & $10,3 \%$ \\
\hline Total & $100,0 \%$ & $100,0 \%$ \\
\hline
\end{tabular}

les eaux d'égout épurées permet la croissance normale d'un peuplement nombreux pendant toute la saison.

Des essais d'hivernage ont été effectués en 1958-1959 avec des alevins de $140 \mathrm{~g}$ et en 1960-1961 avec de petits alevins de $11 \mathrm{~g}$ dans des étangs de la Station expérimentale de pisciculture à Zabieniec, près de Varsovie, alimentés en eau de rivière, ainsi que dans les étangs de Kielce, alimentés en eau d'égout épurée. Des analyses de graisse effectuées sur la chair de ces alevins ont montré le meilleur ètat des alevins conservés dans l'eau d'égout épurée : le taux de graisse atteignait $4 \%$ à Kielce au licu de $1,4 \%$ à Zabieniec.

Des études bactériologiques effectuées simultanément n'ont pas révélé la présence du virus de l'hydropisie infectieuse de la Carpe chez les alevins après l'hivernage. Aucune mort par maladie ou parasite n'a été constatée.

Sur la base des observations et résultats ci-dessus, on peut affirmer :

$1^{\circ}$ Contrairement aux égouts urbains épurés mécaniquement seulement, dont l'usage dans les étangs est restreint à la saison d'été et à condition d'ètre 
dilués au moins 3 à 5 fois avec de l'eau pure, les égouts urbains épurés biologiquement conviennent à l'irrigation d'étangs de pisciculture pendant toute l'année et sans dilution;

20 Les égouts urbains minéralisés forment un milieu riche en nourriture naturelle : une faune nutritive très abondante se développe aux dépens d'un phytoplancton constamment renouvelé, alimenté lui-mème par les composés d'azote, de phosphore et de potassium contenus dans les égouts. Ce milieu s'est montré particulièrement favorable à l'élevage et même à l'hivernage des alevins de Carpe, et, sans fumer les étangs avec des engrais chimiques, on peut produire des quantités importantes de Carpes de deux étés. Cette méthode permettrait de combler le manque d'alevins qui se fait sentir depuis de nombreuses annces dans notre pays;

$3^{0}$ La grande fertilité des égouts minéralisés facilite une forte densité de peuplement : la faune nutritive garantit une croissance continue et préserve des pertes dues à une mauvaise alimentation;

$4^{0} \mathrm{La}$ création d'étangs de pisciculture pour terminer l'épuration des égouts urbains, diminuera la menace de pollution secondaire des rivières et lacs de retenue par les sels minéraux contenus en excès dans ces égouts épurés.

Pawel WOLNY,

Directeur de la Station expérimentale de carpiculture (Ministère de l'Agriculture de Pologne). 